\title{
Emotion Regulation of Neuroticism: Emotional Information Processing Related to Psychosomatic State Evaluated by Electroencephalography and Exact Low-Resolution Brain Electromagnetic Tomography
}

\author{
Shunichiro lkeda Yuko Mizuno-Matsumoto Leonides Canuet \\ Ryouhei Ishii Yasunori Aoki Masahiro Hata Themistoklis Katsimichas \\ Roberto D. Pascual-Marqui Takuto Hayashi Eika Okamoto \\ Tetsuya Asakawa Masao Iwase Masatoshi Takeda
}

\section{Key Words}

a band - Electroencephalography - Emotion .

Exact low-resolution brain electromagnetic tomography .

Functional connectivity · Neuroticism

\begin{abstract}
Emotion regulation is the process that adjusts the type or amount of emotion when we experience an emotional situation. The aim of this study was to reveal quantitative changes in brain activity during emotional information processing related to psychosomatic states and to determine electrophysiological features of neuroticism. Twenty-two healthy subjects (mean age 25 years, 14 males and 8 females) were registered. Electroencephalography (EEG) was measured during an emotional audiovisual memory task under three conditions (neutral, pleasant and unpleasant sessions). We
\end{abstract}

divided the subjects into two groups using the Cornell Medical Index (CMI): (CMI-I: control group, $\mathbf{n = 1 0 : C M I - I I , ~ I I I ~ o r ~ I V : ~}$ neuroticism group, $n=12$ ). We analyzed the digital EEG data using exact low-resolution brain electromagnetic tomography (eLORETA) current source density (CSD) and functional connectivity analysis in several frequency bands $(\delta, \theta, a, \beta, \gamma$ and whole band). In all subjects, bilateral frontal a CSD in the unpleasant session increased compared to the pleasant session, especially in the control group $(p<0.05)$. CSD of the neuroticism group was significantly higher than that of the control group in the full band at the amygdala and inferior temporal gyrus, and in the a band at the right temporal lobe ( $p<0.05$ ). Additionally, we found an increase in functional connectivity between the left insular cortex and right superior temporal gyrus in all subjects during the unpleasant session compared to the pleasant session ( $p<0.05$ ). In this study, using EEG analysis, we could find a novel cortical net- 
work related to brain mechanisms underlying emotion regulation. Overall findings indicate that it is possible to characterize neuroticism electrophysiologically, which may serve as a neurophysiological marker of this personality trait.

\section{Introduction}

Emotional regulation refers to the neuronal processes in the brain that adjust the type or amount of emotion when we experience a particular emotional situation $[1,2]$. Since the deficits of this ability often cause maladjustment and psychopathology, emotion regulation is of paramount importance, especially in the context of a stressful environment. A working model of emotion regulation revealed different cortical regions implicated in the complex cognitive control of emotion. Regions involved in bottom-up emotion generation included mainly the amygdala, whereas top-down processing was associated mainly with prefrontal cortex activity [3-7].

Other neuroimaging studies have also reported that several brain regions are related to emotion regulation. However, the results of these studies are inconsistent. It is well established that the amygdala plays an important role in evoking the emotional response to send incoming sensory information to other brain regions and react appropriately $[8,9]$. In previous studies, limbic regions, including the amygdala, were shown to be deactivated by psychosocial stress [10]. On the other hand, amygdala activity towards negative stimuli has been found to increase after psychosocial stress [11]. It is also said that the prefrontal cortex plays an important role in constructing a complicated cognitive behavior plan, in the expression of personality and in the adjustment of appropriate social actions. In addition, previous studies suggested that induced frontal a activity is a marker of prefrontal cortex activation during an emotional task [12].

Neuroticism is the tendency to be in a negative state and constitutes a fundamental personality trait in psychology. Persons high in neuroticism are those who worry too much, demonstrate high emotional reaction to stress and manifest depression, panic disorder or anxiety neurosis [13]. The extent of neuroticism is generally measured using self-report questionnaires such as the Cornell Medical Index (CMI) and International English MiniMarkers. The CMI is one of the most traditional measures and commonly used in clinical practice. The CMI accurately predicts health status including neuroticism [14].
Quantitative electroencephalography (qEEG) provides computerized imaging and statistical procedures to detect abnormal patterns in specific pathological conditions, and normative features in cognitive and affective disorders [15]. Exact low-resolution brain electromagnetic tomography (eLORETA) is one of the EEG analysis methods that visualizes the origin of brain wave activity obtained from scalp recordings [16]. This method is a discrete, 3D distributed, linear, weighted minimum-norm inverse solution devised by Pascual-Marqui et al. [16]. It illustrates the spread of neural activity with a $7-\mathrm{mm}$ spatial resolution, and, additionally, we can calculate functional connectivity as an index of physiological similarity between brain regions. The particular weights used in eLORETA endow the tomography with the property of exact localization to test point sources, yielding images of current source density (CSD) with exact localization albeit with low spatial resolution. Many studies have demonstrated the validity and reliability of the eLORETA method [17-19].

Measures of linear and nonlinear functional connectivity are implemented in the eLORETA statistical package. eLORETA 'lagged phase synchronization', a nonlinear functional connectivity method, is resistant to some nonphysiological artifacts such as volume conduction and low spatial resolution compared to classic phase synchronization, phase coherence and the imaginary component of coherence [20-22]. The phase lag index suggested by Stam et al. [23] is an improvement in the imaginary part of the coherence as it is less influenced by phase delay. Thus, like eLORETA, this method, which is often used in magnetoencephalography, also helps to control for nonphysiological artifacts.

The aim of this study is to assess quantitative changes during emotional information processing related to psychosomatic states and to determine electrophysiological characteristics of neuroticism.

\section{Subjects and Methods}

Subjects

We recruited 22 healthy adult volunteers ( 14 males and $8 \mathrm{fe}$ males; $22-45$ years old). All subjects gave written informed consent after receiving a detailed explanation of the experimental purposes and research protocol $[24,25]$.

Psychological Tests

Cornell Medical Index

The CMI is a personality test consisting of several questionnaires developed by K. Brodmann at Cornell University in 1949 [26]. The 195 questions that make up the CMI health question- 
Fig. 1. Schematic representation of the emotional audiovisual memory paradigm. EEG was measured under three emotional, audiovisual memory conditions: neutral, pleasant and unpleasant sessions.

\begin{tabular}{|c|c|c|c|c|c|c|}
\hline $120 \mathrm{~s}$ & $40 \mathrm{~s}$ & $180 \mathrm{~s}$ & $40 \mathrm{~s}$ & $180 \mathrm{~s}$ & $40 \mathrm{~s}$ & $180 \mathrm{~s}$ \\
\hline Resting & Viewing & Recalling & Viewing & Recalling & Viewing & Recalling \\
\hline & & $\begin{array}{c}\text { EEG } \\
\text { analysis }\end{array}$ & & $\begin{array}{c}\text { EEG } \\
\text { analysis }\end{array}$ & & $\begin{array}{c}\text { EEG } \\
\text { analysis }\end{array}$ \\
\hline
\end{tabular}

naire are part of a detailed and comprehensive medical interview including the psychological aspects of medical disorders. The CMI is one of the most traditional measures and commonly used in clinical practice. The CMI accurately predicts the health status, including neuroticism [14]. The degree of neuroticism and physical instabilities were assessed in all subjects using the CMI. They were categorized into score I (psychosomatically healthy), II (psychosomatically slightly ill), III (psychosomatically ill) and IV (psychosomatically severely ill, neurotic). We divided the subjects into two groups; the first group included 10 subjects with CMI-I (control group) and the second group consisted of 12 subjects with CMI-II, -III or -IV (neuroticism group).

\section{EEG Recording and Data Acquisition}

EEG recording was performed using a 19-channel Nihon-Kohden Inc. (Tokyo, Japan) system with the electrodes positioned according to the international $10 / 20$ system (i.e. Fp1, Fp2, F3, F4, C3, C4, P3, P4, O1, O2, F7, F8, T7, T8, P7, P8, Fz, Cz and Pz). The EEG was digitized at sampling intervals of $500 \mathrm{~Hz}$, and filtered offline between 1 and $60 \mathrm{~Hz}$.

EEG was measured during an emotional audiovisual memory task using three different conditions: neutral, pleasant and unpleasant sessions. The emotional audiovisual memory task required the subjects to watch some video footages for $40 \mathrm{~s}$ and recall the contents of the videos with their eyes closed. Session 1 used neutral stimuli such as a landscape video. Session 2 used pleasant stimulation such as a comedy video, while session 3 used unpleasant stimuli such as a horror movie (fig. 1).

For each subject, $150 \mathrm{~s}$ of artifact-free EEG data were manually selected. Rejected artifacts included muscle and cardiac contaminations or slow waves related to drowsiness. Further analyses were conducted by the eLORETA-KEY software package.

\section{EEG Source Localization Analysis}

LORETA allows us to compute the source localization of the electric brain activity from scalp EEG data [27-29]. In the current eLORETA package, the solution space is limited to the cortical gray matter, corresponding to 6,239 voxels at $5 \times 5 \times 5 \mathrm{~mm}$ spatial resolution. Electrode coordinates were registered to the Montreal Neurologic Institute average MRI brain (MNI152) [30]. We performed a CSD analysis in several frequency bands $(\delta: 2-4 \mathrm{~Hz}$, $\theta: 4.5-7.5 \mathrm{~Hz}, \alpha: 8-13 \mathrm{~Hz}, \beta: 13.5-30 \mathrm{~Hz}, \gamma: 30.5-60 \mathrm{~Hz}$ and full band: $2-60 \mathrm{~Hz}$ ).

\section{Functional Connectivity Analysis}

For functional connectivity analysis, we created regions of interest, selecting all 42 Brodmann areas in each hemisphere provided in the eLORETA software. Linear and nonlinear lagged phase synchronization between pairs of Brodmann areas was used as a measure of functional connectivity $[23,29,31$, 32]. Lagged phase synchronization measures the similarity of these signals in the frequency domain based on normalized (unit module) Fourier transforms. It is thought to contain only physiological connectivity information because it represents the connectivity between two signals after the instantaneous zerolag contribution has been excluded. In fact, zero-lag connectivity is sensitive to nonphysiological artifacts such as volume conduction $[33,34]$. The lagged phase synchronization value was calculated between all pairs of regions of interest (861 connections).

\section{Statistical Analyses}

We compared the value of CSD and functional connectivity provided by eLORETA between the neuroticism group and the control group for each frequency band. For statistical neuroimaging analysis, eLORETA applies a statistical nonparametric mapping method [34], and statistical differences between groups are assessed using Student's t test (based on Fisher's permutation method; threshold at the $5 \%$ probability level). In addition, to correct for multiple comparisons, a permutation test using 5,000 data randomizations was carried out.

\section{Results}

\section{Source Localization}

We found an increase in a CSD in the frontal cortex bilaterally during the unpleasant session compared with the pleasant session. This difference was statistically significant in the control group $(\mathrm{p}<0.05)$. CSDs in the full band at the amygdala and inferior temporal gyrus were significantly higher in the neuroticism group compared with the control group. In addition, the neuroticism group also exhibited an increase in a CSD at the right temporal lobe $(p<0.05)$. No other CSD was statistically significant (fig. 2, 3).

\section{Functional Connectivity}

We found a functional connectivity increase between the left insular cortex and right superior temporal gyrus in the unpleasant session compared with the pleasant session, specifically in the $a$ band $(p<0.05$; fig. 4$)$. Linear and 
Fig. 2. Results of CSD analysis (unpleasant vs. pleasant sessions) in the $\alpha$ band. In all subjects, bilateral frontal a CSD in unpleasant sessions increased compared with pleasant sessions, especially in the control group $(\mathrm{p}<0.05)$.

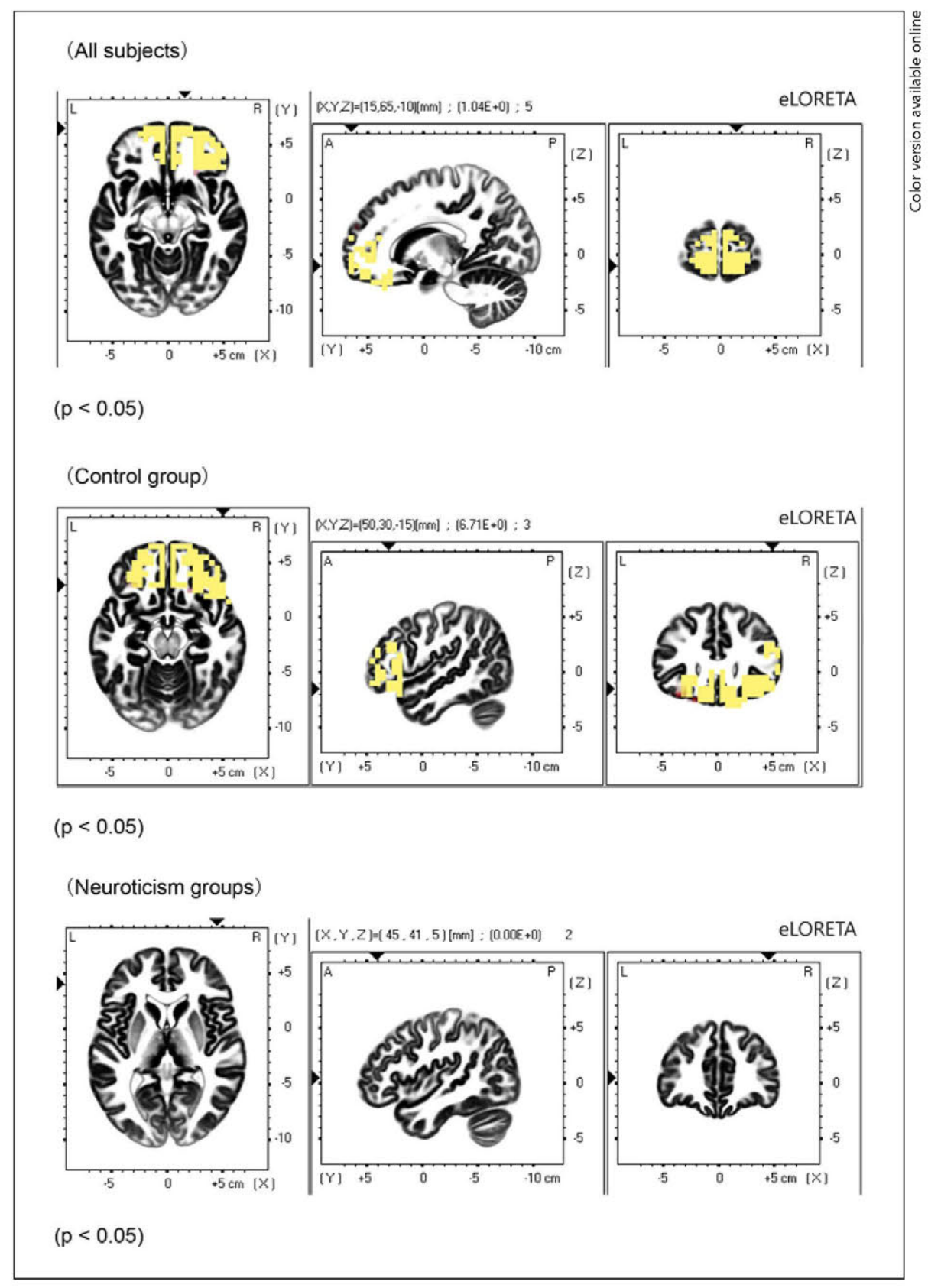

nonlinear lagged phase synchronization showed exactly the same connectivity (fig. 4). There were no significant differences between other groups.

\section{Discussion}

In the present study, we used CSD and physiological linear and nonlinear connectivity analyses to identify quantitative changes in cortical activity during emotional information processing related to psychosomatic states and to clarify electrophysiological characteristics of neuroticism. We found increased bilateral frontal a CSD in the unpleasant session compared to the pleasant session in the control group. In the neuroticism group, we found increased CSD in the full band at the amygdala and inferior temporal gyrus, as well as in the right temporal lobe for the a band.

Several studies have reported that $\alpha$ power in the frontal lobe increased following unpleasant stimula- 


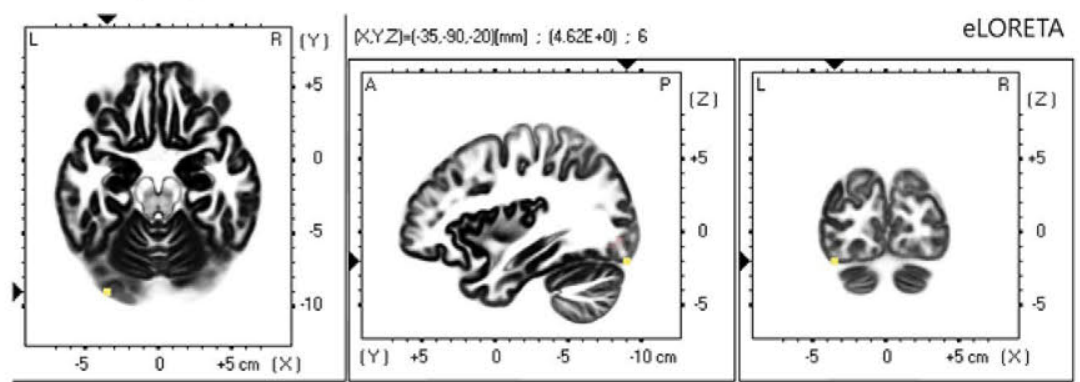

(Neuroticism groups)

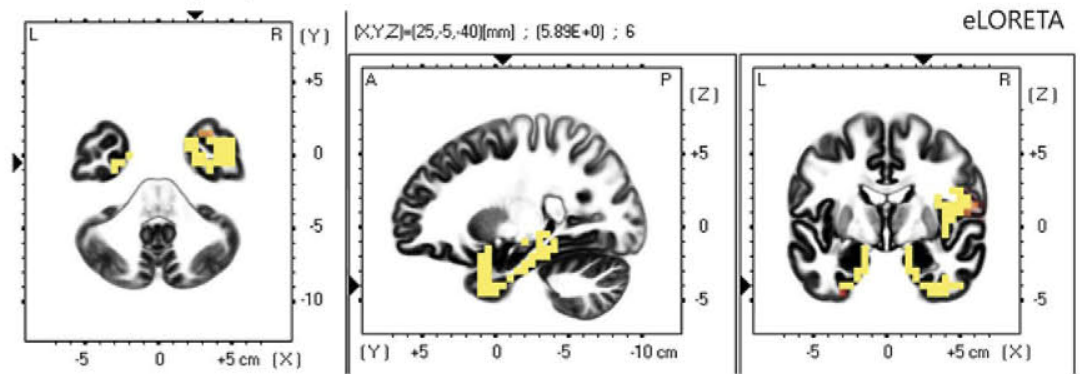

Unpleasant session vs Neutral session ( $\alpha$ band)

(Control group)

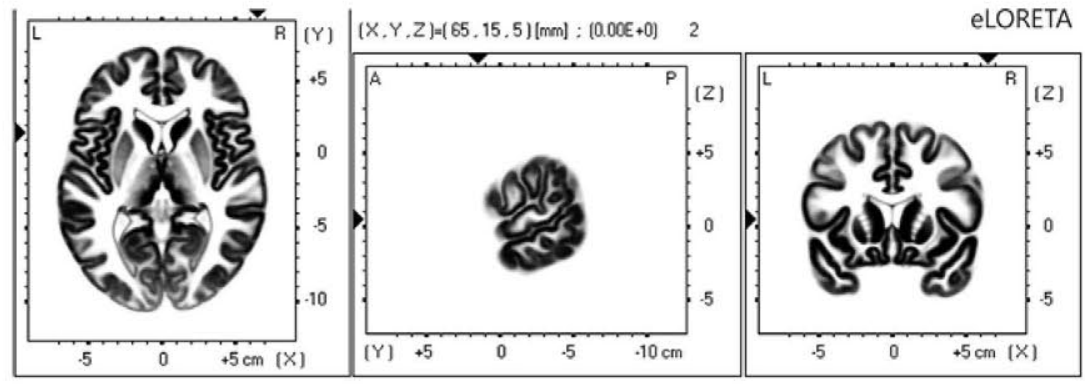

(Neuroticism group)

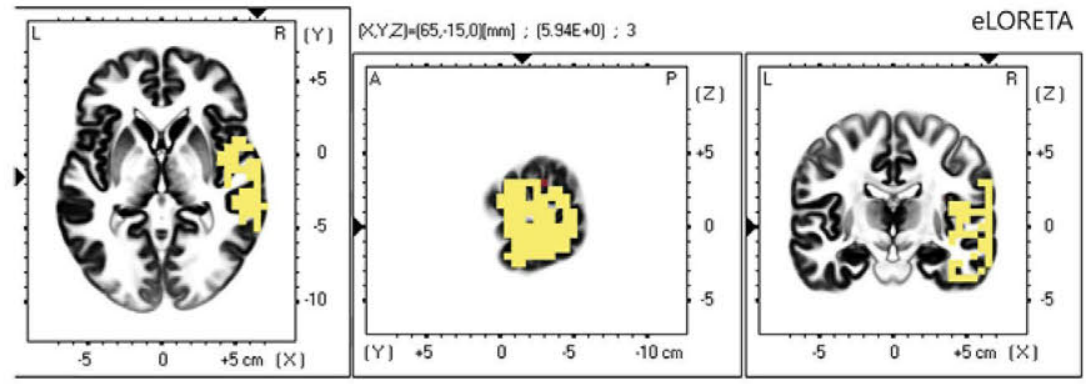

Fig. 3. Results of CSD analysis (unpleasant vs. neutral sessions). CSD of the neuroticism group was significantly higher than that of the control group in the full band at the amygdala and the inferior temporal gyrus, and in the $\alpha$ band at the right temporal lobe $(\mathrm{p}<0.05)$.
$5+5 \mathrm{~cm}(\mathrm{X})$ 
Fig. 4. Results of connectivity analysis. a Left lateral view. b Coronal view from the front. c Axial view from the top. The significant connection (between the left insula and the right superior temporal lobe) is projected onto a transparent fiducial cortical surface. The points to which the lines are connected represent the center of the Brodmann area mass. The red color (colors refer to the online version only) of the line indicates increased connectivity in the neuroticism group relative to the control group.

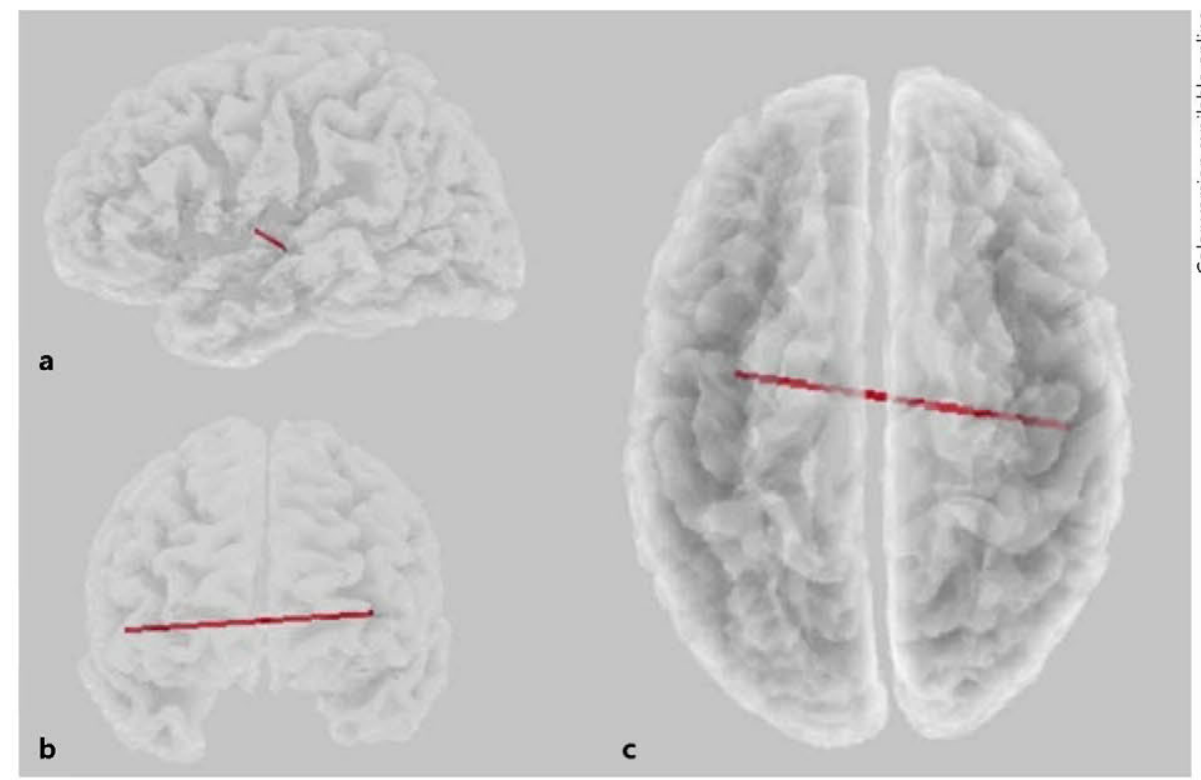

tion. Based on those studies, frontal a oscillation may be related to cortical inhibition. In line with this view, reduced a power has been associated with increased prefrontal cortex activity during working memory performance [35-39]. Viviani et al. [40] suggested that unpleasant emotional stimuli reduce the activity in frontal executive attention areas, including the dorsolateral prefrontal cortex. Thus, our results suggest that control subjects used spontaneous emotion regulation in the prefrontal cortex during unpleasant stimuli.

The functional roles of the amygdala and prefrontal brain regions in emotional processing have already been reported in several studies. The amygdala receives input during negative events from cortical brain regions, particularly the medial prefrontal cortex [8]. This region is thought to be related to emotional conflicts and regulation of autonomic and affective responses during fear conditioning. The left amygdala has been linked to social anxiety, obsessive compulsive disorder and posttraumatic stress [41]. Other studies have reported that hyperactivity in the amygdala was observed when patients saw unpleasant stimuli such as threatening faces [42]. They noted that the more severe the general anxiety disorder the greater the response in the amygdala observed in the patients.

Further, we found a functional connectivity increase between the left insular cortex and the right superior temporal gyrus during unpleasant stimuli compared to pleasant stimuli in the a band. The insular cortex is an important multisensory integration area and mediates interpretation of sensory information from the body. This area is also involved in emotion regulation, visceral sensory perception, self-awareness, cognitive functioning and shared feeling. Particularly, the anterior insular cortex is reported to be associated with emotional feelings such as anger, fear, sadness and happiness [43]. On the other hand, the posterior insula is thought to be associated with auditory visual function [44]. The insula sends information to other limbic brain regions such as the amygdala, the ventral striatum and the orbitofrontal cortex [45]. The superior temporal gyrus is home to the primary auditory cortex for sound perception as well as the region for language comprehension and processing [46]. In the present study, we found that functional connectivity analysis suggests there is a link in information processing between the superior temporal gyrus and the insular cortex.

Some limitations of this study are acknowledged. First, unfortunately, the sample size was small. A larger study cohort might provide more significant results. However, based on the properties of lagged phase synchronization and because correction for multiple comparisons was performed, the findings obtained are thought to reflect true physiological connectivity and CSD. Secondly, the two groups included in our study (individuals with neuroticism and controls) are both healthy subjects. Investigation in neurosis patients might be able to clarify whether these connectivity changes represent state or trait markers of neurosis. Third, LORETA has low spatial resolution. Although this might make it difficult to visualize the activity 
of really small and deep structures, such as the amygdala, Moont et al. [47] reported activation of the amygdala in their EEG study about the conditioned pain modulation effect using LORETA analysis. We suggest that LORETA also could depict CSD in the amygdala in our study.

In summary, through the use of eLORETA analysis, we found a novel cortical network likely related to brain mechanisms underlying emotion regulation. This finding suggests that it is possible to characterize neuroticism electrophysiologically, which may serve as a neurophysiological marker of this personality trait. Future studies are needed to shed more light on this important matter.

\section{Acknowledgments}

This research was partially supported by the Center of Innovation Program from the Japanese Science and Technology Agency.

\section{References}

1 Gross JJ: The emerging field of emotion regulation: an integrative review. Rev Gen Psychol 1998;2:271-299.

2 Sripada C, Angstadt M, Kessler D, Phan KL, Liberzon I, Evans GW, Welsh R, Kim P, Swain JE: Volitional regulation of emotions produces distributed alterations in connectivity between visual, attention control, and default networks. NeuroImage 2014;89:110-121.

3 Viviani R: Emotion regulation, attention to emotion, and the ventral attentional network. Front Hum Neurosci 2013;7:746.

4 Ochsner KN, Bunge SA, Gross JJ, Gabrieli JDE: Rethinking feelings: an FMRI study of the cognitive regulation of emotion. J Cogn Neurosci 2002;14:1215-1229.

5 Miller EK: The prefrontal cortex and cognitive control. Nat Rev Neurosci 2000;1:5965.

6 Ochsner KN, Gross JJ: The cognitive control of emotion. Trends Cogn Sci 2005;9:242249.

7 Ochsner KN, Knierim K, Ludlow DH, Hanelin J, Ramachandran T, Glover G, Mackey SC: Reflecting upon feelings: an fMRI study of neural systems supporting the attribution of emotion to self and other. J Cogn Neurosci 2004; 16:1746-1772.

8 LeDoux JE: Emotion circuits in the brain. Annu Rev Neurosci 2000;23:155-184.

9 Phillips ML, Drevets WC, Rauch SL, Lane R: Neurobiology of emotion perception I: the neural basis of normal emotion perception. Biol Psychiatry 2003;54:504-514.

10 Pruessner JC, Dedovic K, Khalili-Mahani N, Engert V, Pruessner M, Buss C, Renwick R, Dagher A, Meaney MJ, Lupien S: Deactivation of the limbic system during acute psychosocial stress: evidence from positron emission tomography and functional magnetic resonance imaging studies. Biol Psychiatry 2008; 63:234-240.

11 Oei NY, Veer IM, Wolf OT, Spinhoven P, Rombouts SA, Elzinga BM: Stress shifts brain activation towards ventral 'affective' areas during emotional distraction. Soc Cogn Affect Neurosci 2012;7:403-412.

12 Parvaz MA, MacNamara A, Goldstein RZ, Hajcak G: Event-related induced frontal al- pha as a marker of lateral prefrontal cortex activation during cognitive reappraisal. Cogn Affect Behav Neurosci 2012;12:730-740.

13 Hettema JM, Neale MC, Myers JM, Prescott CA, Kendler KS: A population-based twin study of the relationship between neuroticism and internalizing disorders. Am J Psychiatry 2006;163:857-864.

14 Pendleton N, Clague JE, Horan MA, Rabbitt PM, Jones M, Coward R, Lowe C, McInnes $\mathrm{L}$ : Concordance of Cornell medical index self-reports to structured clinical assessment for the identification of physical health status. Arch Gerontol Geriatr 2004;38:261269.

15 Bjørk MH, Sand T, Bråthen G, Linaker OM, Morken G, Nilsen BM, Vaaler AE: Quantitative EEG findings in patients with acute, brief depression combined with other fluctuating psychiatric symptoms: a controlled study from an acute psychiatric department. BMC Psychiatry 2008;8:89.

16 Pascual-Marqui RD, Michel CM, Lehmann D: Low resolution electromagnetic tomography. A new method for localizing electrical activity in the brain. Int J Psychophysiol 1994; 18:49-65.

17 Corsi-Cabrera M, Galindo-Vilchis L, del-RíoPortilla Y, Arce C, Ramos-Loyo J: Withinsubject reliability and inter-session stability of EEG power and coherent activity in women evaluated monthly over nine months. Clin Neurophysiol 2007;118:9-21.

18 Cannon RL, Baldwin DR, Shaw TL, Diloreto DJ, Phillips SM, Scruggs AM, Riehl TC: Reliability of quantitative EEG (qEEG) measures and LORETA current source density at 30 days. Neurosci Lett 2012;518:27-31.

19 Canuet L, Ishii R, Pascual-Marqui RD, Iwase M, Kurimoto R, Aoki Y, Ikeda S, Takahashi H, Nakahachi T, Takeda M: Resting-state EEG source localization and functional connectivity in schizophrenia-like psychosis of epilepsy. PLoS One 2011;6:e27863.

20 Worsley KJ, Chen JI, Lerch J, Evans AC: Comparing functional connectivity via thresholding correlations and singular value decomposition. Philos Trans R Soc Lond B Biol Sci 2005;360:913-920.
21 Canuet $L$, Ishii $R$, Iwase $M$, Kurimoto $R$, Ikezawa $\mathrm{K}$, Azechi $\mathrm{M}$, Wataya-Kaneda $\mathrm{M}$, Takeda M: Tuberous sclerosis: localizing the epileptogenic tuber with synthetic aperture magnetometry with excess kurtosis analysis. J Clin Neurosci 2008;15:1296-1298.

22 Schoffelen JM, Gross J: Source connectivity analysis with MEG and EEG. Hum Brain Mapp 2009;30:1857-1865.

23 Stam CJ, Nolte G, Daffertshofer A: Phase lag index: assessment of functional connectivity from multi channel EEG and MEG with diminished bias from common sources. Hum Brain Mapp 2007;28:1178-1193.

24 Okamoto E, Hayashi T, Nishimura $\mathrm{H}$, Inada H, Ishii R, Ukai S, Shinosaki K, Mizuno-Matsumoto Y: EEG activities evoked by trauma stimuli related to earthquakes and personality features associated with trauma. Int J Intell Comput Med Sci Image Process 2011;4:13 24.

25 Mizuno-Matsumoto $\mathrm{Y}$, Hayashi T, Okamoto E, Asakawa T, Sawamura K, Ishii R, Ukai S, Shinosaki K: Measurement of personality stability in infants and young adults under emotional stimuli using a brain functional reaction method. Int J Intell Comput Med Sci Image Process 2011;4:39-64.

26 Gibson HB, Hanson R, West DJ: A questionnaire measure of neuroticism using a shortened scale derived from the Cornell Medical Index. Br J Soc Clin Psychol 1967;6:129-136.

27 Pascual-Marqui RD: Discrete, 3D distributed, linear imaging methods of electric neuronal activity. Part 1: exact, zero error localization. arXiv:0710.3341. http://arxiv.org/ pdf/0710.3341.

28 Pascual-Marqui RD: Theory of the EEG inverse problem; in Tong $\mathrm{S}$, Thakor $\mathrm{N}$ (eds): Quantitative EEG Analysis: Methods and Applications. Boston, Artech House, 2009, pp 121-140.

29 Pascual-Marqui RD, Lehmann D, Koukkou M, Kochi K, Anderer P, Saletu B, Tanaka H, Hirata K, John ER, Prichep L, Biscay-Lirio R, Kinoshita T: Assessing interactions in the brain with exact low-resolution electromagnetic tomography. Phil Trans A Math Phys Eng Sci 2011;369:3768-3784. 
30 Mazziotta J, Toga A, Evans A, Fox P, Lancaster J, Zilles K, Woods R, Paus T, Simpson G, Pike B, Holmes C, Collins L, Thompson P, MacDonald D, Iacoboni $\mathrm{M}$, Schormann $\mathrm{T}$, Amunts K, Palomero-Gallagher N, Geyer S, Parsons L, Narr K, Kabani N, Le Goualher G, Boomsma D, Cannon T, Kawashima R, Mazoyer B: A probabilistic atlas and reference system for the human brain: International Consortium for Brain Mapping (ICBM). Philos Trans R Soc Lond B Biol Sci 2001;356: 1293-1322.

31 Pascual-Marqui RD: Instantaneous and lagged measurements of linear and nonlinear dependence between groups of multivariate time series: frequency decomposition. arXiv: 0711.1455. http://arxiv.org/abs/0711.1455.

32 Mulert C, Kirsch V, Pascual-Marqui R, McCarley RW, Spencer KM: Long-range synchrony of $\gamma$ oscillations and auditory hallucination symptoms in schizophrenia. Int J Psychophysiol 2011;79:55-63.

33 Nolte G, Bai O, Wheaton L, Mari Z, Vorbach $\mathrm{S}$, Hallett M: Identifying true brain interaction from EEG data using the imaginary part of coherency. Clin Neurophysiol 2004;115: 2292-2307.

34 Liang M, Zhou Y, Jiang T, Liu Z, Tian L, Liu $\mathrm{H}, \mathrm{Hao} \mathrm{Y}$ : Widespread functional disconnectivity in schizophrenia with resting-state functional magnetic resonance imaging. Neuroreport 2006;17:209-213.
35 Klimesch W: EEG alpha and theta oscillations reflect cognitive and memory performance: a review and analysis. Brain Res Rev 1999;29: 169-195.

36 Klimesch W, Doppelmayr M, Rohm D, Pollhuber D, Stadler W: Simultaneous desynchronization and synchronization of different alpha responses in the human electroencephalograph: a neglected paradox? Neurosci Lett 2000;284:97-100.

37 Klimesch W, Sauseng P, Hanslmayr S: EEG alpha oscillations: the inhibition-timing hypothesis. Brain Res Rev 2007;53:63-88.

38 Knyazev GG: Motivation, emotion, and their inhibitory control mirrored in brain oscillations. Neurosci Biobehav Rev 2007;31:377 395.

39 Park CA, Kwon RJ, Kim SHJ, Chae JH, Kim $\mathrm{T}$ : Decreased phase synchronization of the EEG in patients with major depressive disorder. World Congr Med Phys Biomed Eng 2006, pp 1095-1098.

40 Viviani R, Lo H, Sim E-J, Beschoner P, Stingl JC, Horn AB: The neural substrate of positive bias in spontaneous emotional processing. PLoS One 2010;5:e15454.
41 Moses-Kolko EL, Perlman SB, Wisner KL, James J, Saul AT, Phillips ML: Abnormally reduced dorsomedial prefrontal cortical activity and effective connectivity with amygdala in response to negative emotional faces in postpartum depression. Am J Psychiatry 2010; 167:1373-1380.

42 Phan KL, Fitzgerald DA, Nathan PJ, Tancer ME: Association between amygdala hyperactivity to harsh faces and severity of social anxiety in generalized social phobia. Biol Psychiatry 2006;59:424-429.

43 Craig AD: How do you feel - now? The anterior insula and human awareness. Nat Rev Neurosci 2009;10:59-70.

44 Hashimoto T, Taoka M, Obayashi S, Hara Y, Tanaka M, Iriki A: Modulation of cortical vestibular processing by somatosensory inputs in the posterior insula. Brain Inj 2013;27:16851691.

45 Craig AD: A new view of pain as a homeostatic emotion. Trends Neurosci 2003;26: 303-307.

46 Bigler ED, Mortensen S, Neeley ES, Ozonoff S, Krasny L, Johnson M, Lu J, Provencal SL, McMahon W, Lainhart JE: Superior temporal gyrus, language function, and autism. Dev Neuropsychol 2007;31:217-238.

47 Moont R, Crispel Y, Lev R, Pud D, Yarnitsky D: Temporal changes in cortical activation during conditioned pain modulation (CPM), a LORETA study. Pain 2011;152:1469-1477. 